\title{
On Reproduction of Fuzziness in C-E Poetry Translation
}

\author{
Guihua Cao \\ Foreign Language School, Xiaogan University, Xiaogan 432000, China \\ E-mail: gillcao@163.com
}

\begin{abstract}
John Turner, an outstanding Chinese culture researcher, once commented that Chinese culture is the most artistic and longest-established culture that ever existed. Poetry is the succinct part in Chinese and culture-loaded. Fuzziness is one of basic features of poetry, especially in Chinese classical poetry. Fuzzy language is widely used in Chinese classical poetry, and its translation has become a difficult task because of differences between Chinese and English. The author of this paper begin with the theory of fuzziness and explore the reproduction of fuzzy language.
\end{abstract}

Keywords: Artistic conception, Chinese classical poetry, Fuzziness

\section{Fuziness in peotry}

V.G. Belinskiy(1811-1848) once said, "emotions are one of vital dynamic factors in the nature of poetry. No emotions, no poets, and no poems". The nature of peotry is to issue the authors' emtions. Rich emotions and complexity decide fuziness in poety.

Fuzziness refers to the indefiniteness and impreciseness of language. It is a communicative device and it is the feature of literary works. Without fuzziness, language will lose its nature as a social phenomenon. In Webster's Ninth New Collegiate Dictionary (P. 500), fuzziness is defined as "Lacking in clarity of definition." Fuzziness has at least three features: indeterminacy, relativity and shift from accuracy to fuzziness. In 1902, an American philosopher, mathematician and writer, gave a proper definition. "When many possibilities may occur and the speaker can't decide whether he should remove this possibility out of the definition or not even though he thinks carefully about these possibilities, we think this definition fuzzy." (Quoted in fuzzy language by Wu Tieping:136)

In 1984, Liu Fu published his paper titled On the Fuzziness and Determinacy of Character/ Personality. In his paper, he expressed that a radical distinction between literature and science lied in the fact that we can use numbers to describe science while in literature we can only use aesthetic language such as images, feelings, plot etc. That is why literature is fuzzy. Fuzziness is the basic nature of literature. It makes poems and artistic conception meaningful. In fuziness theory, fuzziness is considered the basical feature and common phenomenon in the world. Distincness is relative. Fuziness in peotry means meaning of the peotry is undefinite. Poetry is used to show our feelings. But besides distinct love and hatred, there is something between the two. In peotry, Language is employed to show poets' feelings. Compared accurate language with fuzzy language, the latter is more charming.

Fuzziness is nearly everywhere in the ancient Chinese poetry, such as "qianli jiangling yiri huan", "putao meijiu yeguang bei", "ren bi huanghua shou", "guopo shanhe zai, chengchun caomu shen"In these lines, there is fuzy language. "qianli","yiri"are accurate, while here is fuzy. The first line means the boat travels fast and the the peot is very delighted. And the other three are also fuzzy. What kind of good wine is it? What kind of lininous wine glass? To what degree the country is damaged? How long the grass is? The fuzy language leave the readers space to imagine. The line, "ren bi huanghua shou", gives us more sapce to imagine. To what degree the grass is slim? Slimmer than chrysanthemum. Then to what degree the chrysanthemum is slim? From these examples, we can see that fuzzy language in pqotry is more propriate. Immanual Kant(1924-1804) said, "fuzzy definition can display more accurate language, ...beauty is something that can not be expressed."

Fuzziness is one of basical features of poetry, esp. in ancient Chinese peory. Fuziness makes the powms more open, and adds depth and width, and leave more space for readers to imagine. Fuzziness is indeterminate and relative. Fuzziness can be identified as semantic fuzziness, image fuzziness, syntactic fuzziness, pragmatic fuzziness, and thematic fuzziness.

Fuzziness leaves literary gaps to readers and gives readers more space to imagine. Literary gaps are caused by semantic, image, pragmatic, syntactic and thematic indeterminacy. This indeterminacy forms appealing structure to encourage readers to think and imagine. The extremely excellent production of artistic conception is fuzzy beauty. Fuzziness contributes to artistic conception. 


\section{Poetry translation}

The ancient Chinese peotry values implicateness, beautiful, vigor of style, atomasphere, spitits and etc. Fuziness is obvious in peotry. As readers, we should try our best to understand the fuzziness in peotry; as tranlsators, we must first figure out the meaning of the poetry, and then try to convey the fuzziness and artistic conception.

As to the criteria of peotry translation, many scholars gave their ideas, such as Alexander F. Tytler's (1747----1814) "three principles" in 1790, Eugene.A. Nida's, a famous American linguistist, dynamic equivalence and Functional Equivalence in his later years and so on. In China, Yan Fu advocated faithfulness, expressiveness and elegance, while Qu Qiubai, Mao Dun thought translators should keep the style of the original and present content and form completely and correctly. Lin Yutang considered "faithfulness, expressiveness and beauty" as the criteria. Xu Yuanzhong thought that poetry translation should be as beautiful as the original in sense, in sound and, if possible, in form. There are many criteria for poetry translation. No matter what criteria it is, artistic conception and faithfulness or equivalence are admitted by all of the them. However, absolute faithfulness or equivalence can never be achieved. It is just an ideal. What translators can do is try to make the translation as close as possible to the original, and make the translation achieve the same aesthetic effect on target readers as the original does on the source readers.

\section{Interpreting fuzzy language}

The American peot, Robert Frost said, "Poetry is that which is lost in translation."

Since fuzziness is one of basical features of poetry, different readers with different experience and other factors will have different understanding. Imagination can hardly be controlled. And Chinese and English belong to different language family. All these factors makes ancient Chinese peotry difficult to be translated into English. Even so, many people worked hard and presented us many excerllent translations.

Peotry employs definite words to express rich meaning. Fuzzy language is usually employed in Chinese poetry. But fuzziness is seldom used in English. English needs subjects, verbs, articles, etc. So when Chinese poetry is translated into English, translators try to find out the most proper way to interpret the original, to convey the meaning and to reproduce artistic conception. There are many ways to convey fuziness, such as interpretion and finding out corresponding fuzzy language in target language.

No matter how hard translators tried, lose of fuzziness is unavoidable. As translators, we can only try to make the readers of original and tranlsation have the same or similar aesthetic experience.

Sematic fuzziness is often used in literature, and together with ellipsis dots. In peotry, it is seldom used, so semantic fuzziness will not be discussed in this paper.

(1) To syntactic fuzziness, we should make the sentence complete but not tedious.

In some poems, the poets ommitted the position in order to make the poems brief. In this way, syntactic fuzziness appears. It will not cause misunderstanding of the original readers. But if it is translated word for word into English, the translation will be inappropriate In English speaking countries. So, in oder to avoid misunderstanding, we should make the sentence complete. Let's look some examples.

A. Kuteng laoshu hunya From Ma Zhiyuan's Tianjingsha.qiusi

English version:

Withered vines hanging on old branches,

Returning crow croaking at dusk. .

By Ding Yinzu and Burnton Raffel

B. Damo guyan zhi, cahnghe luori yuan. From Wang Wei's shi zhi saishang

English version:

Straight is the lonely line of smoke above the desert vast,

And round, the sun that sets upon the long river.

By Zhang Tingchen and Wei Bosi

C. Zhongting dibai shu qi ya, lenglu wusheng shi guihua.

From Wang Jian"s Missing Mr. Du on seeing the moon

English version:

In the moonlight courtyard crows are nesting in the trees;

Osmanthus flowers soundlessly are dampened by the dew.

By Zhang Tingchen and Wei Bosi

In the above three examples, the relation between images are not indicated in the original. But in English translations, 
the prepostion are given. If we ommitted the prepositions as the original, the lines will be simply images presentation, which is not appropriate in English.

In brief, prepostions should be given in English translation to make the translation acceptable according to the language habits and convey the artistic conception well.

(2) To image and thematic fuzziness, we should keep it fuzzy on the basis of full understanding

In Chinese, there are many fuzzy languages, such as "three", "six", "nine", "twelve", etc. These figures neednot be translated as accurate numbers. We can employ corresponding fuzzy language in the target language. Because of the differences between English and Chinese, there are seldom plural and singular form in Chinese. When transalting, we must pay attention to the understanding and translation of these images and translate them according to our understanding. Take Liu Zongyuan's jaingxue as an example. In the poem, jiangxue, there are lines"qianshan niao fei jue, wanjing ren zong mie." Here, "qian", "wan"donot mean there are one thousand birds and ten thousand roads, no more, no less. They mean many, lots of. When transalting, we can use "hundreds of"and"thousands of" in translation.

There are more exmples.

A. Shijian xingle yi ruci, gulai wanshi dongliushui. From Libai's mengyou tianmu yin liubie

Throughout all time all things are as the waters,

Flowing to the sea.

So too is earthly pleasure.

By Zhang Tingchen and Wei Bosi

B. Zhifou? Zhifou? Yingshi lvfeihongshou. Fom Li Qingzhao's Rumengling

I cry, “Can't you see? Can't you see?

\section{The green leaves are flesh but the red flowers are fading.}

By Dai Naidie and Yang Xianyi

Form these two examples, we know that when we deal with fuzzy language, we must first understand the meaning of the peoms; after we know what the peot wants to say and what emotions he/she wants to express, we can decide which word will best express the peot's emotions and convey the artistic coneption When translating Rumengling, Dai Naidie and Yang Xianyi translated " $l v$ "into "green leaves", and "hong" into "red flowers"according to their understanding.

In the opinion of the author of this paper, the transaltion are excellent.

Transation does not mean only passive conveyance. The more important is that it means active creation. If you want to translate peotry, you must first be a poet.

\section{Conclusion}

Inter-culture communication is possible because people admit that there exist shared cultural phychology and thing mode between nations. When something is conveyed to another culture, something surely will be lost. Chinese is a fuzzy language, while English tends to be more accurate. So loss is unavoidable in dealing with the ancient Chinese poetry characterized as fuzziness. And so is difficulty. Nbo matter what method we adopt, we should try to keep the original meaning and convey the artistic conception faithfully.

\section{References}

Dai Naidie, Yang Xianyi el.. (2001). Chinese-English Ancient Peotry:Songci. Beijing:Foreign language press.

Essay on the Principles of Translation. London: J.M. Dent and Co.

Eugene. A. Nida. (1964). Toward a Science of Translating with Special Reference to Principles and Procedures Involved in Bible Translating. Leiden: E.J. Bill. 159,166----175 and 183

Guo, Zhuzhang \& Li, Qingsheng. (2003).Practical English-Chinese Translation Course. Wuhan:Wuhan University Press.

New Literature and Art. (1958). Literature in Belinskiy's mind. 14

Xu Jun. (2003).On Translation. Wuhan:Hubei Education Press.

$\mathrm{Xu}$, yuanchong, Lu, Peixuan \& Wu, Juntao. (1997).The selected 300 Tangshi and its English Translation. Beijing:External translation Press Company.6, $5^{\text {th }}$ version.

Yan, Yu. Canglang Shihua-On Poetry.

Yang, Chuanwei. (1992). American peoms selections. Beijing:Beijing Normal University. P114

Zhang, Tingchen \& Wei, Bosi. (1997). The selected 100 Tangshi and its English Translation. Beijing:External translation Press Company.6. $5^{\text {th }}$ version. 